\title{
The optimisation, design and verification of feed horn structures for future Cosmic Microwave Background missions
}

\author{
Darragh McCarthy ${ }^{\mathrm{a}, *}$, Neil Trappe ${ }^{\mathrm{a}}$, J. Anthony Murphy ${ }^{\mathrm{a}}$, Créidhe O’Sullivan ${ }^{\mathrm{a}}$, Marcin Gradziel ${ }^{\mathrm{a}}$, \\ Stephen Doherty ${ }^{\mathrm{a}}$, Peter G. Huggard ${ }^{\mathrm{b}}$, Arturo Polegro ${ }^{\mathrm{c}}$, Maarten van der Vorst ${ }^{\mathrm{c}}$ \\ ${ }^{a}$ Department of Experimental Physics, Maynooth University, Maynooth, Kildare, Ireland \\ ${ }^{\mathrm{b}}$ Rutherford Appleton Laboratory, Harwell Campus, Didcot OX11 0QX, United Kingdom \\ ${ }^{\mathrm{c}}$ ESA ESTEC, Keplerlaan 1, 2201 AZ Noordwijk, Netherlands
}

\section{H I G H L I G H T S}

- Efficient mode-matching based optimisation process for feed horns is presented.

- Allows design of high performance smooth-walled feed horns using desktop computers.

- Horn designed for future CMB missions with rigorous performance requirements.

- Horn meets all requirements across the band, only taking $6.5 \mathrm{~h}$ to design.

- Horn was manufactured and measurements agreed excellently with simulations.

\section{A R T I C L E I N F O}

\section{Article history:}

Received 8 December 2015

Available online 28 January 2016

\section{Keywords:}

Horn antennas

Antenna design

Millimeter wave antennas

Optimisation

Genetic algorithm

Cosmic Microwave Background

\begin{abstract}
A B S T R A C T
In order to investigate the origins of the Universe, it is necessary to carry out full sky surveys of the temperature and polarisation of the Cosmic Microwave Background (CMB) radiation, the remnant of the Big Bang. Missions such as COBE and Planck have previously mapped the CMB temperature, however in order to further constrain evolutionary and inflationary models, it is necessary to measure the polarisation of the CMB with greater accuracy and sensitivity than before.

Missions undertaking such observations require large arrays of feed horn antennas to feed the detector arrays. Corrugated horns provide the best performance, however owing to the large number required (circa 5000 in the case of the proposed COrE+ mission), such horns are prohibitive in terms of thermal, mechanical and cost limitations.

In this paper we consider the optimisation of an alternative smooth-walled piecewise conical profiled horn, using the mode-matching technique alongside a genetic algorithm. The technique is optimised to return a suitable design using efficient modelling software and standard desktop computing power. A design is presented showing a directional beam pattern and low levels of return loss, cross-polar power and sidelobes, as required by future $\mathrm{CMB}$ missions. This design is manufactured and the measured results compared with simulation, showing excellent agreement and meeting the required performance criteria.

The optimisation process described here is robust and can be applied to many other applications where specific performance characteristics are required, with the user simply defining the beam requirements.
\end{abstract}

(c) 2016 Elsevier B.V. All rights reserved.

\section{Introduction}

The measurement of full sky maps of the Cosmic Microwave Background (CMB) radiation, the remnant of the Big Bang, is critical in the fields of astrophysics and cosmology. Polarisation sensitive measurements of this radiation allow for the detailed study of the origins of the Universe and the structures contained within it,

\footnotetext{
* Corresponding author. Tel.: +35317083552.

E-mail address: darragh.mccarthy@nuim.ie (D. McCarthy).
}

allowing further constraints to be placed on the various inflationary and evolutionary models that currently exist. Many missions have recorded such full sky maps, for example COBE [1], WMAP [2] and more recently Planck [3]. In order to continue progressing in these areas, it is necessary to design instruments which are polarisation sensitive as this will allow the measurement of the CMB E- and B-modes, revealing additional information centred around $100-150 \mathrm{GHz}$, about the various inflationary models.

Such high performance polarisation sensitive instruments can potentially make use of several array formats, including horns, bare 
arrays and lens arrays. Horn arrays are popular as they are proven technology, have low stray light coupling and do not require the use of a cold stop to truncate sidelobe structure or even define the beam. Such arrays typically make use of corrugated feed horn antennas to feed the detector array. In order to increase the sensitivity, it is necessary to increase the number of pixels on the focal plane, and so this makes the use of traditional corrugated horns prohibitive due to more complex cryogenics and manufacturing processes and associated cost implications. A suitable alternative to the use of corrugated horns would be the use of a smooth walled horn which is optimised to retain the high performance of a corrugated horn in terms of the figures of merit which are of interest for CMB missions, at the expense of those which are less critical. For example, a typical requirement for a CMB mission to measure polarisation would be low cross-polar power, high beam symmetry and low return loss in order to maximise the throughput of the measured signal, so these performance metrics could be optimised.

Additionally, it is necessary to be flexible in the design process of such horns, being able to change the horn geometry to meet any new requirements that arise from constraints associated with the optics or the focal plane. A lengthy optimisation would hinder this process, as would the need to be able to access a supercomputer at short notice. To this end, it is desirable that the optimisation process be capable of completion within a reasonable timeframe and using only the standard desktop computing power which is typically available. Rapid horn optimisation also helps in providing a robust and efficient optics design process. Although future CMB missions were chosen as the example with which to demonstrate the optimisation process, it can be applied to any application whose performance requirements are known, including multimode systems.

The optimisation of feed horn antennas is a problem which has received considerable attention [4-7]. A particular aim of the investigation presented in this paper is to maximise the efficiency of the optimisation process, using only standard desktop computing power to achieve the particular design criteria for the system in question. This allows a simple horn geometry to be realised quickly and efficiently when compared to some other optimisation techniques where large supercomputers are required. In order to achieve this goal, it was necessary to adapt a more efficient approach in terms of the complexity of the optimisation process relative to those presented in the references above, and to see how well a horn resulting from this simple process could approach the required high levels of performance in terms of the performance metrics of interest, in this case those required by future CMB missions. In this approach, the horn geometry is defined in terms of $N$ segments, where $N$ is a relatively small number in order to reduce the computational effort required during the optimisation process but allowing sufficient degrees of freedom to achieve good performance.

Unlike in the investigations undertaken by other groups where these segments are profiled by interpolation using a spline that represents some function, such as $\sin ^{2}$ for example, here the segments are linearly interpolated. This reduces the computational complexity of the problem, however as the profile is now less smooth it becomes more difficult to achieve the required level of performance, and so it is of interest to investigate whether the required performance (high beam symmetry, low levels of crosspolarisation power return loss and sidelobe levels) can be realised using this simpler design. We refer to this type of horn geometry as a piecewise conical profile horn ( $\mathrm{PCPH})$, a design envisaged to have sufficient degrees of freedom to achieve the required performance. This type of feed horn geometry is outlined in [8], along with an analysis of the impact of using the geometry along with various figures of merit and constraints in the optimisation process.

\section{Optimisation process}

In this section the optimisation process is described, including a description of the mode-matching technique which is used to simulate waveguide structures. The modifications necessary to make the mode-matching technique more efficient in order to allow the overarching optimisation process to converge are also described, in addition to a description of the integration of the mode-matching technique with the optimisation algorithm and the resulting horn design.

\subsection{The mode-matching technique}

To simulate the performance of a waveguide horn antenna, code developed in-house known as SCATTER [9], based on the modematching technique as applied to a two port system [10], is used. SCATTER allows the calculation of the transmission and reflection scattering matrices ( $\mathrm{S}$ matrices) which govern the bulk behaviour of the antenna, giving a full vector description of its performance. Results predicted using SCATTER have been extensively verified against experimental results for both single and multi-mode systems and so it can be applied to the analysis of waveguide structures with confidence.

In the mode-matching technique, the field in the waveguide structure is represented by a combination of transverse electric (TE) and transverse magnetic (TM) modes, with the scattering matrices that result from the analysis representing the complex coefficients of these modes in reflection and transmission. Using these scattering matrices the field patterns of the horn (co-polar, cross-polar and unpolarised) can be calculated at the aperture or in the far field, along with many associated figures of merit such as reflected and cross-polar power, and beam symmetry.

In any iterative optimisation process that uses the modematching technique to calculate the figure of merit, it is necessary to execute SCATTER a large number of times. It is therefore necessary to make SCATTER as computationally efficient as is possible in order to minimise the computational time and resources required for the optimisation process. By using only the required number of modes at each junction the scattering matrix calculation step is already very efficient, however the field pattern calculation can be time consuming. This is an issue, as the field pattern must be accurately calculated in order to determine many figures of merit in terms of the performance of the horn. The modification made to the calculation in order to improve the speed of this step will now be described.

Often, when the transmission matrix at the aperture of the horn is analysed (the $S_{21}$ matrix in the convention used in SCATTER), many of the modes are found to not contribute significantly to the field. For example in a single-mode horn, as is being considered here, there will only be one channel of power supported by the horn. The unsupported modes are still included in the calculation, resulting in an increase in computational time. It is not advisable to simply ignore these modes as their contribution, however small, is real. It is possible however to reduce the execution time by expressing the output basis set of the horn in a more efficient manner by using the mathematical technique of singular value decomposition (SVD) [11].

If $\mathbf{S}$ is an $m \times n$ matrix representing the $S_{21}$ matrix, then the SVD of $\mathbf{S}$ is defined as

$\mathbf{S}=\mathbf{U} \cdot \boldsymbol{\Sigma} \cdot \mathbf{V}^{\dagger}$

where $\mathbf{U}$ is an $m \times m$ unitary matrix, $\boldsymbol{\Sigma}$ is an $m \times n$ rectangular matrix containing non-negative real numbers on the diagonal, and zeros elsewhere, and $\mathbf{V}^{\dagger}$, the complex transpose of $\mathbf{V}$, is an $n \times n$ 
unitary matrix. The diagonal entries of $\boldsymbol{\Sigma}, \Sigma_{i i}$, are known as the singular values of $\mathbf{S}, \sigma_{i}$, and are arranged in order of decreasing value. The columns of $\mathbf{U}$ form a basis set for the modes supported at the aperture of the horn, when scaled by their corresponding singular values, according to $\sigma_{i} U_{i}$. The magnitude of the singular value represents the fraction of the indicated mode that propagates from the input of the system to the output. Due to this, it is clear that only modes with a non-zero singular value are present at the aperture, and so these are the natural modes supported by the system. Due to the diagonal nature of $\boldsymbol{\Sigma}$, scattering can only occur between the $i$ th columns of $\mathbf{U}$ and $\mathbf{V}$ (the basis set for the input modes of the system). This means that the modes are incoherent and propagate independently, and so when summing over the modes to calculate the field at some plane they must be added in quadrature.

The modes with non-zero singular values are the true modes supported by the horn, with the power contained in each mode determined by $\sigma_{i}^{2}$. Adding the supported modes in quadrature allows the aperture or far field beam patterns of the horn to be found with no compromise in accuracy, as shown in Fig. 1. As modes which do not contribute are no longer included, this results in significant time savings. In the example shown in Fig. 1 the horn (standard corrugated horn at $100 \mathrm{GHz}$ ) is single-mode and using the SVD method results in only one mode being considered during the field calculations, as opposed to 30 . The 'original' calculation took $171.26 \mathrm{~s}$ and the SVD based method took $\approx 3.1 \mathrm{~s}$, again showing the increase in computational efficiency with no compromise in accuracy. The computer used in this simulation and all others presented in this paper was a Dell Optiplex 780 PC with an Intel Core 2 duo $2.93 \mathrm{GHz}$ processor, operating Windows 7 and with 3 GB RAM.

\subsection{Genetic algorithm based optimisation process}

SCATTER was altered to include the modifications outlined previously, and integrated with the standard genetic algorithm optimisation routine that available for MATLAB as a part of the Global Optimisation Toolbox. The genetic algorithm [12] is a global optimisation method that is based on natural selection. It can be readily applied to multi-variable problems such as those encountered in horn optimisation. Each 'generation' of the algorithm corresponds to various sets of values for the optimisation parameters (children) being used to evaluate the figure of merit. When all sets have been evaluated, the ones giving the lowest value for the figure of merit become "parents" and are combined in various ways to produce the children which populate the next generation.

The piecewise conical profile horn was chosen as the basis for the optimisation process, with the optimisation variables being the length and radius of each section (6 sections), thus effectively varying the flare angle of each section. MATLAB allows upper and lower limits to be placed on these variables, meaning that the overall physical dimensions of the horn can be controlled. For example, specifying upper and lower limits for the radius of the final section allows the maximum and minimum values for the aperture radius to be determined. This is critical for controlling modal content and also for ensuring that the horns will fit in a densely packed focal plane array, as is the case for future CMB missions which these horns could be used in. These limits also allow control over the length of the horn, which again is a critical design parameter.

For CMB applications, it was found by observation that minimising the cross-polar level gave the best performance in terms of meeting all other critical performance requirements, and so this was chosen as the figure of merit. This figure of merit shall be referred to as the cross/co-polar ratio. Each iteration of the genetic algorithm corresponds to a unique set of values for the input parameters, and so corresponds to a unique horn design being evaluated. In each iteration, the co- and cross-polar fields are found

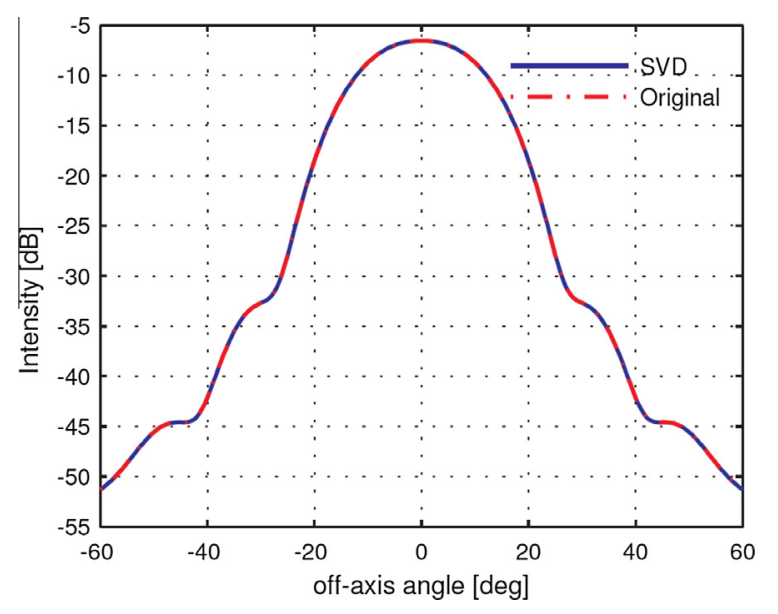

Fig. 1. Comparison of far field pattern ( $E$ plane) obtained using both the 'original' and SVD based field pattern calculations for a standard corrugated horn at $100 \mathrm{GHz}$.

using the mode-matching technique, and the cross/co-polar ratio is passed to the optimiser, allowing it to vary the input parameter values and iterate towards a design which minimises this figure.

Table 1 shows the upper and lower bounds and the optimised values for the optimisation parameters used to define the horn, with Fig. 2 showing a profile view of the final optimised design. The optimisation was carried out at $100 \mathrm{GHz}$, which is the centre of the desired $85-115 \mathrm{GHz}$ band of operation. A spot frequency was used to reduce the computational time and to observe if the target of a $10 \%$ bandwidth could be realised using only a simple spot frequency optimisation. Only the $n=1$ azimuthal order was considered, as the horn is to be used in single-mode, WR10 waveguide fed operation. The algorithm took $6.5 \mathrm{~h}$ to iterate to the indicated solution despite the number of variables, which meets the requirement that the design process can be carried out using a standard desktop computer within a reasonable timeframe.

\section{Measurement of fabricated horn}

The optimised horn design was manufactured by the STFC Rutherford Appleton Laboratory (RAL) in the United Kingdom. The complete feed included a single step transition which matched the circular geometry waveguide of the throat of the horn to rectangular WR10 waveguide. This is to facilitate testing with a vector network analyser (VNA), as the VNA interfaces via WR10 waveguide. This transition ensures that the horn supports one true mode of propagation, even though modes with $n \neq 0$ are supported by the horn (this allowed modes with only $n=1$ to be considered in the design process). The complex transmission matrices were measured by means of a Rohde and Schwarz ZVA - 24 VNA used in a two port configuration, with two ZV - 110 converter heads used to extend its range to the W-band (75-110 GHz). A full TOSM (through, offset, short, match) calibration was also carried out.

Table 1

Optimisation parameters. Lower and upper refer to the bounds placed on each parameter to specify envelope of horn dimensions. Final is the optimised value for each parameter.

\begin{tabular}{llcc}
\hline Parameter & Lower $[\mathbf{m m}]$ & Upper $[\mathbf{m m}]$ & Final $[\mathbf{m m}]$ \\
\hline Length & 8.000 & 10.000 & 8.781 \\
Radius 1 & 1.200 & 2.000 & 1.604 \\
Radius 2 & 1.600 & 4.000 & 3.417 \\
Radius 3 & 3.400 & 6.000 & 3.528 \\
Radius 4 & 5.500 & 8.000 & 5.694 \\
Radius 5 & 7.500 & 10.000 & 7.837 \\
Radius 6 & 9.500 & 13.000 & 12.969 \\
\hline
\end{tabular}




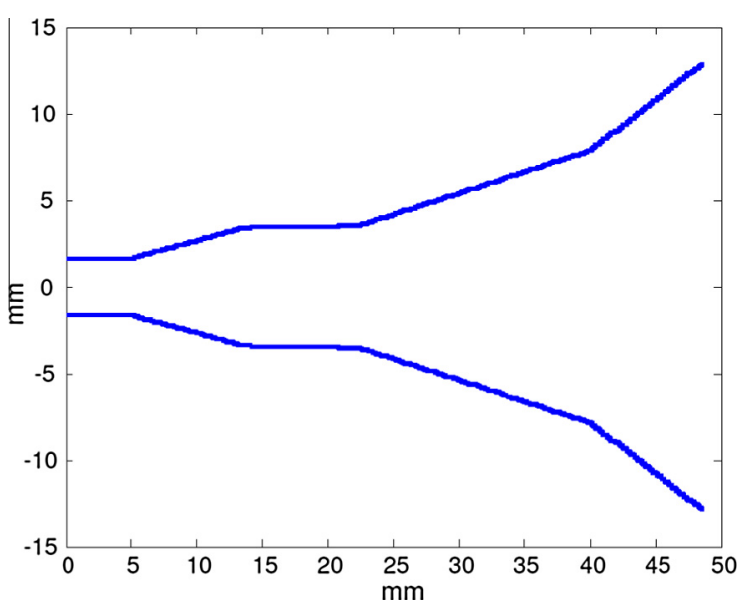

Fig. 2. Profile view of the optimised horn design (based on a design frequency of $100 \mathrm{GHz}$ ).

The horn was mounted on one frequency converter head and fixed to a $z$-translation stage, with the other head mounted on an $x y$ raster scanner to allow a planar scan of the complex near-field of the horn at different distances from the horn aperture. As the converter head acting as the detector presents a bare waveguide to free space, a WR10 waveguide probe is mounted on the converter head in order to minimise the reflections which would otherwise occur. At the frequencies in question, this results in only free space power that couples to the fundamental linearly polarised $\mathrm{TE}_{10}$ mode being detected. This means that polarisation sensitive measurements of the horn are possible. With careful alignment (using a Gaussian beam fitting routine to maximise component placement), suppression of reflections and calibration, an effective dynamic range of $\approx 80 \mathrm{~dB}$ was realised across the band with a $100 \mathrm{~Hz}$ measurement bandwidth. The translation stage was used to vary the detector to horn aperture separation from 40 to $340 \mathrm{~mm}$.

An overview of the measured field patterns can be found in [13], however a thorough analysis of the measurement campaign will now be given here. Figs. 3 and 4 respectively show the co- and cross-polar fields of the horn having propagated $40 \mathrm{~mm}$, at a frequency of $100 \mathrm{GHz}$. The co-polar beam is highly symmetric, showing low sidelobe levels. The symmetry is quantified in Fig. 5, which gives the coupling between orthogonal cuts of the beam across the band. The quad lobe structure in the cross-polar field is also clearly visible. This beam quality is maintained at all frequencies, indicating excellent bandwidth performance of both the horn and measurement system, with the required levels of symmetry and sidelobe levels being met.
The measurement system was simulated using the in-house developed optical modelling package, Maynooth Optical Design and Analysis Laboratory (MODAL) [14]. This also uses the modematching technique to simulate the horn antenna and then uses physical optics to propagate the beam from the aperture to the measurement plane. Here, the waveguide probe was included in the model (by convolving the field from the horn with the $\mathrm{TE}_{10}$ field of the probe) along with the ability to include offsets and rotations about the horn axes. It is also possible to include the raster scanning regime in the simulation. Including these effects, the measured data show excellent agreement with simulation. Fig. 6 shows a comparison of two equivalent cuts through the simulated and measured co-polar beams at $100 \mathrm{GHz}$, showing the high level of agreement. The same level of agreement is observed across the band, providing excellent validation of both the simulation software and the measurement testbed. Measurements were taken over the 75-110 GHz VNA operating band only.

In terms of cross-polar performance, the cross/co-polar ratio improves for higher frequencies, in line with simulated trends, both of which are shown in Fig. 7 with similar performance observed at all distances. The levels measured average $\approx 3 \mathrm{~dB}$ higher than the simulated figures across the band due to the presence of the transition, which is not included in the simulations. This increases return loss (see Fig. 8), which results in increased cross-polar levels. The only major disagreement between the measured and simulated levels occurs at $100 \mathrm{GHz}$. The predicted level (in the absence of the transition) is extremely low $(\approx-45 \mathrm{~dB})$, and so to measure this, particularly in the presence of the transition, is extremely difficult. It is therefore not surprising to note a difference between simulated and measured values when the simulated value is extremely low. The horn design is also heavily customised for $100 \mathrm{GHz}$ operation, and so although the predicted figures at this frequency are undoubtedly real, they are based on an ideal system with no misalignments, no transition and a circular waveguide as the source. Any deviations from this, which of course exist in real systems, will result in the performance deviating from the ideal case. This is clearly shown in Fig. 7 , where the performance at $100 \mathrm{GHz}$ is clearly a departure from the overall trend. The measured value is still very low and so the actual performance at $100 \mathrm{GHz}$ is acceptable.

Such performance across a wide band is ambitious for a smooth-walled horn that was optimised at a spot frequency. For future $\mathrm{CMB}$ missions such as COrE+ which will use large arrays of feed horns (the application for which this horn is being developed), a $10 \%$ bandwidth (95-105 GHz), derived from the science requirements, is proposed for the various $\mathrm{CMB}$ channels. Applying this to the horn and centring the band on $100 \mathrm{GHz}$ shows that the cross/co-polar ratio remains below $-25 \mathrm{~dB}$ across this band, broadly achieving the target levels.
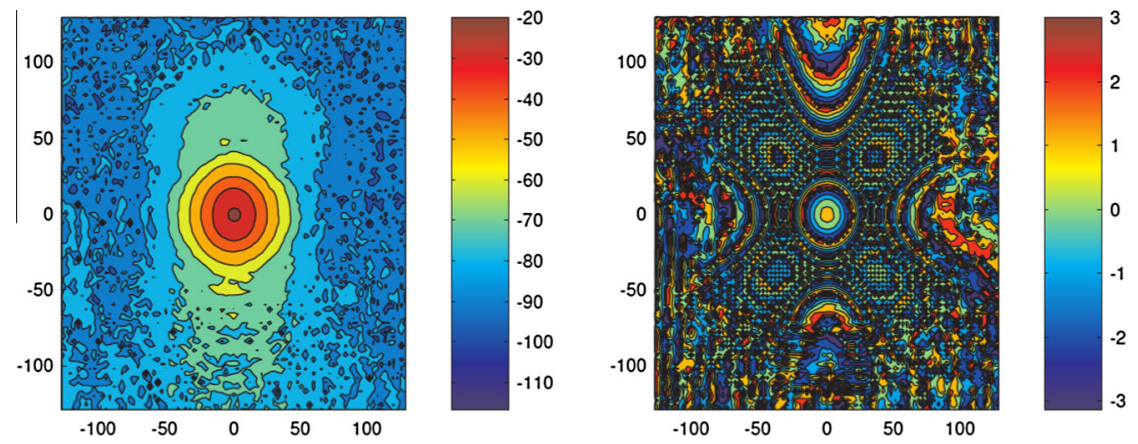

Fig. 3. Co-polar intensity (left, units of $\mathrm{dB}$ ) and phase (right, units of radian) for the optimised horn, measured $40 \mathrm{~mm}$ from the aperture. Distances in units of mm. 

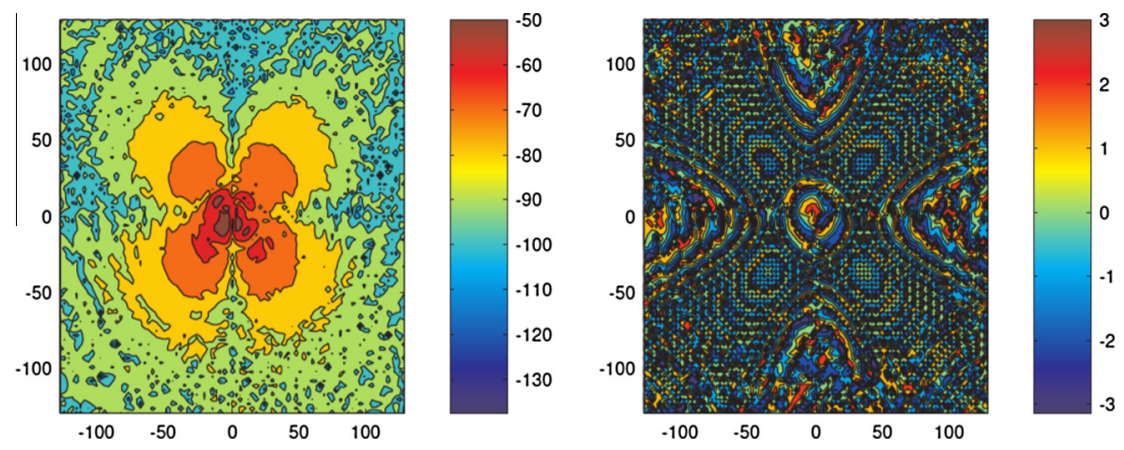

Fig. 4. Cross-polar intensity (left) and phase (right), measured $40 \mathrm{~mm}$ from the aperture.

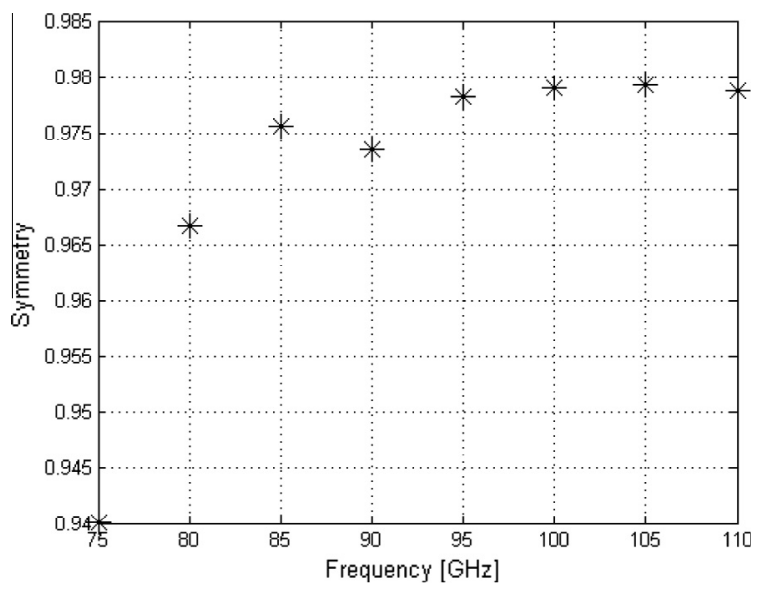

Fig. 5. Coupling between orthogonal cuts of the measured co-polar pattern across the band.

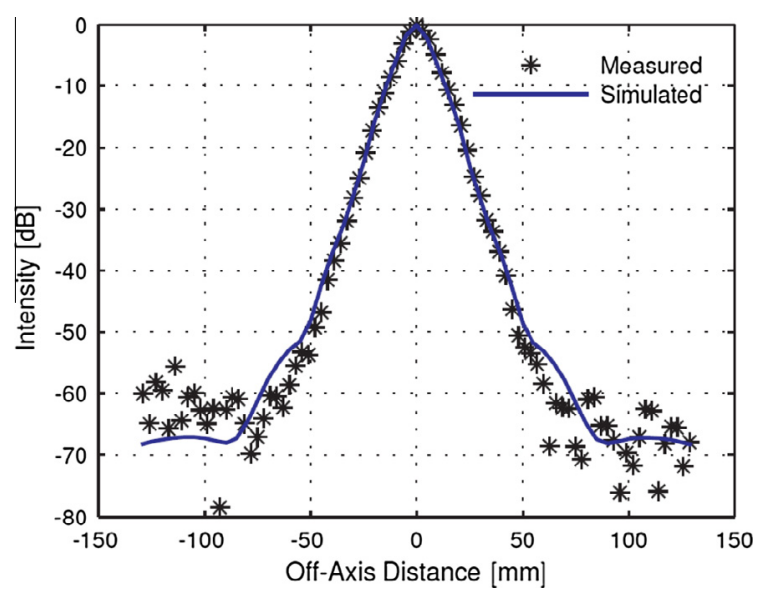

Fig. 6. Comparison of measured and simulated co-polar fields at $100 \mathrm{GHz}$, at a distance of $40 \mathrm{~mm}$.

The return loss across the band is shown in Fig. 8. SCATTER, which does not include the transition, predicts lower levels than either CST or the measurements which both include the transition. This is expected, as the transition introduces reflections to the system, increasing $S_{11}$. The CST results show an increase in the levels of return loss as the transition is now included and so more reflections are present in the system. This is consistent with the transition being responsible for the increase in cross-polar levels. The measured return loss is slightly higher than the levels predicted by CST, likely due to misalignments within the measurement system giving rise to increased scattering in addition to that caused

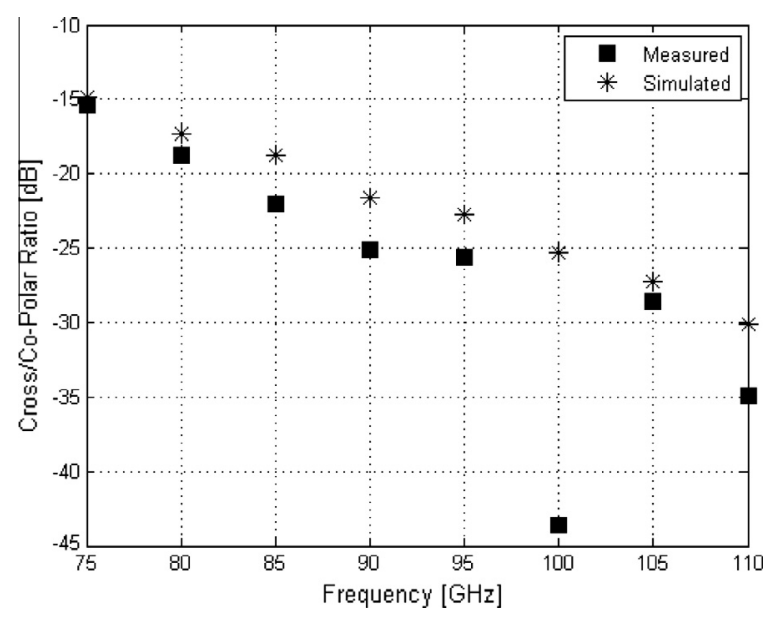

Fig. 7. Comparison of measured and simulated (including imperfections) crosspolar ratios at $100 \mathrm{GHz}$ and a distance of $40 \mathrm{~mm}$.

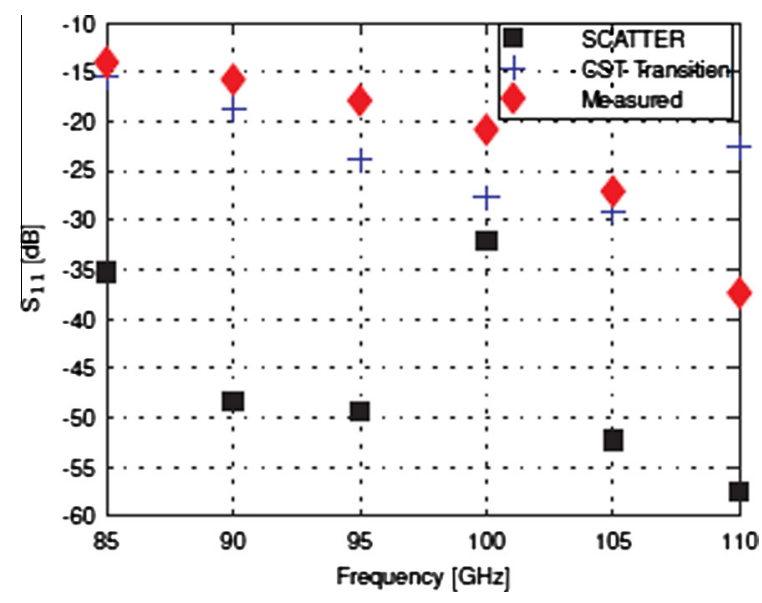

Fig. 8. Simulated and measured return loss $\left(S_{11}\right)$ across the band.

by the transition. The CST model assumes perfect alignment and so would not account for the increased return loss due to this.

\section{Conclusion}

For thermal, mechanical and cost reasons, it is necessary to develop high performance alternatives to corrugated horns for use in future $\mathrm{CMB}$ missions where large (thousands of pixels), densely packed arrays will be employed. Such horns are difficult to design using standard commercially available tools due to the computational complexity and time requirements. This is 
troublesome when such instruments are being designed and a change occurs in the optical/focal plane design, requiring a relatively quick redesign of the horn antennas in order that the system can be re-evaluated.

In this paper we illustrated how the mode-matching technique can be modified and combined with a genetic algorithm to form an efficient optimisation tool. The principal modification to the technique involved the use of singular value decomposition to represent the output basis set of the horn antenna in a more efficient manner, reducing the complexity of the field calculation. Additionally, the code was altered to consider a variable number of modes at each junction within the structure. These modifications resulted in significant time savings, making the mode-matching technique more computationally efficient.

Using a simple piecewise conical profile horn as the basis for the optimisation process, this tool was used to design a smooth-walled horn of simple geometry that largely meets the requirements of future CMB missions across the likely bandwidths that will be used (10\%). The six section horn was manufactured by RAL, and its performance verified experimentally, showing excellent agreement with simulated results and meeting the various performance requirements, despite being designed at a single frequency and also including a rectangular to circular transition to match to WR10 waveguide.

These results show that it is possible to use a very simple basis design and optimisation tool in order to design a smooth-walled conical profile horn that largely meets the requirements of future CMB missions without having to make use of corrugated horns. Although the application chosen to demonstrate the design principle was that of a future CMB mission, the process is robust and so readily lends itself to designing horn antennas for a wide variety of other applications, including multi-mode systems where the increase in efficiency offered by this approach is particularly advantageous. All that is required is that the performance criteria for the application are well known, and a suitable horn antenna can be designed.

\section{Conflict of interest}

There is no conflict of interest.

\section{Acknowledgements}

The authors would like to acknowledge funding from the European Space Agency under the Strategic Initiative for Irish Industry,
Academia and Research Institutes, and the Irish Research Council (formerly IRCSET) under the EMBARK scheme.

\section{Appendix A. Supplementary material}

Supplementary data associated with this article can be found, in the online version, at http://dx.doi.org/10.1016/j.infrared.2015.12. 014.

\section{References}

[1] C. Bennett, N. Boggess, E. Chang, M. Hauser, et al., Scientific results from cobe, Adv. Space Res. 12 (12) (1993) 409-423.

[2] C. Bennett et al., Nine-year wilkinson microwave anisotropy probe (wmap) observations: final maps and results, Astrophys. J. Suppl. Ser. 208 (2013) 20.

[3] The Planck Collaboration, Planck 2015 results. I. Overview of products and scientific results, Astron. Astrophys. (2015).

[4] C. Granet, G. James, A smooth-walled spline-profile horn as an alternative to the corrugated horn for wide band millimetre-wave applications, IEEE Trans. Antennas Propag. 52 (3) (2004).

[5] C. Granet, G. James, R. Bolton, G. Moorey, Smooth-walled spline-profile horn for radio astronomy, in: Proc. JINA 2002 Int. Symp. Antennas, Nice, France, 2002.

[6] C. Granet, T. Bird, Optimization of corrugated horn radiation patterns via a spline-profile, in: Proc. ANTEM '02, Montreal, Quebec, 2002.

[7] G. Yassin, P. Kittara, A. Jiralucksanawong, S. Wangsuya, J. Leech, M. Jones, A high performance horn for large format focal plane arrays, in: Proc. 18th International Symposium on Space Terahertz Technology, California Institute of Technology, Pasadena, 2007.

[8] D. McCarthy, N. Trappe, J. Murphy, et al, Efficient horn antennas for nextgeneration terahertz and millimeter-wave space telescopes, in: SPIE Proceedings 8624, Terahertz, RF, Millimeter and Submillimeter-Wave Technology and Applications VI, San Francisco, 2013.

[9] R. Colgan, J. Murphy, B. Maffei, C. O’Sullivan, R. Wylde, P. Ade, Modelling fewmoded horns for far-ir space applications, in: Proc. 11th International Symposium on Space Terahertz Technology, Ann Arbor, Michigan, 2000, pp. $368-378$.

[10] A. Olver, P. Clarricoats, A. Kishk, L. Shafai, Microwave Horns and Feeds, IEEE, 1994.

[11] S. Withington, M. Hobson, R. Berry, Representing the behaviour of partially coherent optical systems by using overcomplete basis sets, J. Opt. Soc. Am. 21 (2) (2004)

[12] K. Man, K. Tang, S. Kwong, Genetic algorithms: concepts and applications, IEEE Trans. Ind. Electron. 43 (5) (1996).

[13] D. McCarthy, N. Trappe, J.A. Murphy, et al., Efficient algorithms for optimising the optical performance of profiled smooth walled horns for future $\mathrm{cmb}$ and far-ir missions, in: SPIE Proceedings 9153, Millimeter, Submillimeter, and FarInfrared Detectors and Instrumentation for Astronomy VII, Montreal, Quebec, 2014.

[14] M. Gradziel, C. O'Sullivan, J. Murphy, et al., Modelling of the optical performance of millimeter-wave instruments in modal, in: SPIE Proceedings, terahertz and Gigahertz Electronics and Photonics VI, 2007. 